\title{
Perspectivas para o desenvolvimento dos cultivos de camarões marinhos no estuário da Lagoa dos Patos, RS
}

\author{
Perspectives for the development of marine shrimp farming in the estuary of \\ Patos Lagoon, RS, Brazil \\ Luís Poersch $^{1}$ Ronaldo Oliveira Cavalli ${ }^{2}$ Wilson Wasielesky Júnior ${ }^{3}$ \\ Jorge Pablo Castello ${ }^{4}$ Silvio R. M. Peixoto ${ }^{4}$
}

\section{- REVISÃO BIBLIOGRÁFICA -}

\section{RESUMO}

A produção brasileira de camarões vem apresentando um crescimento significativo nos últimos anos, concentrando-se na região nordeste, principal pólo produtor do país. Muito embora as condições climáticas da região Sul não permitam a produção durante todo o ano, uma quantidade considerável de investimentos vem sendo aplicada. No estuário da Lagoa dos Patos, RS, os cultivos da espécie nativa Farfantepenaeus paulensis em cercados e gaiolas $e$ de Litopenaeus vannamei em viveiros estão sendo desenvolvidos como novas atividades, as quais certamente poderão favorecer o desenvolvimento social e econômico da região. Entretanto, a implantação desordenada da carcinicultura pode gerar sérios problemas ambientais. $O$ presente trabalho apresenta uma visão das características ambientais da região em questão e descreve os sistemas de cultivo atualmente utilizados. Além disso, as principais dificuldades enfrentadas por esta nova atividade, assim como a discussão de uma legislação adequada às condições locais $e$ a definição de áreas apropriadas para o cultivo, são apresentadas. Finalmente, o desenvolvimento sustentável da carcinicultura na região é discutido sob as perspectivas social, econômica e ambiental.

Palavras-chave: cultivo, camarão marinho, Rio Grande do Sul, sustentabilidade.

\begin{abstract}
Shrimp production in Brazil has presented a significant increase in the last few years, especially in the northeastern region. Although climatic conditions in southern Brazil do not allow year-round production, considerable investments have been made in the region. In the estuarine area of Patos Lagoon, the culture of the native Farfantepenaeus paulensis in cages and pen enclosures, and of Litopenaeus vannamei in earthen ponds are being developed as new activities that will certainly produce social and economical development to the region. Nevertheless, the implantation of shrimp farming without proper ordination and control may generate serious environmental problems. The present paper presents an overview of the environmental characteristics of the area and describes the farming systems currently adopted. Furthermore, the main challenges faced by this new activity, such as the discussion of a legislation adapted to local conditions, and the definition of areas appropriate for farming are presented. Finally, the sustainable development of shrimp farming is discussed under social, economical and environmental perspectives.
\end{abstract}

Key words: cultive, marine shrimp, Rio Grande do Sul, sustentability.

\footnotetext{
${ }^{1}$ Departamento de Oceanografia, Fundação Universidade Federal do Rio Grande (FURG), Estação Marinha de Aquacultura, CP 474, 96201-900, Rio Grande, RS, Brasil. E-mail: lpoersch@mikrus.com.br.

${ }^{2}$ Departamento de Oceanografia, FURG, Estação Marinha de Aquacultura, CP 474, 96201-900, Rio Grande, RS, Brasil. E-mail: cavalli@mikrus.com.br

${ }^{3}$ Departamento de Oceanografia, FURG, Estação Marinha de Aquacultura, CP 474, 96201-900, Rio Grande, RS, Brasil E-mail: manow@mikrus.com.br

${ }^{4}$ Departamento de Oceanografia, FURG, Estação Marinha de Aquacultura, CP 474, 96201-900, Rio Grande, RS, Brasil E-mail: docjpc@furg.br
} 


\section{INTRODUÇÃO}

Em nível mundial, o cultivo de camarão marinho teve seu crescimento acelerado nas duas últimas décadas, aumentando de 215.000 toneladas em 1985 para 865.000 toneladas no ano 2000, o que representa $43 \%$ do total produzido em todo o mundo (FAO, 2002).

No Brasil, observa-se tendência semelhante ao que aconteceu em outros países, como China, Tailândia, Vietnã, Índia, Indonésia e Bangladesh, que hoje dominam o mercado mundial da carcinicultura. $\mathrm{O}$ clima favorável do Brasil, somado ao domínio das novas tecnologias de produção, colocam o país como o principal produtor de camarões das Américas. Entre 1997 e 2001, a produção de camarões marinhos cultivados no Brasil cresceu a uma taxa anual de cerca de $83 \%$, passando de 3.600 toneladas para 40.000 toneladas/ano ao final de 2001. Entre 2002 e 2003, a produção de camarões cultivados apresentou um crescimento anual de 50\%, alcançando valores superiores a 90.000 toneladas/ano (ABCC, 2004).

Na região Sul, em função das temperaturas mais amenas, o cultivo de camarões marinhos esteve direcionado para espécies nativas, em função da adaptação a estas condições. Entretanto, a falta de um pacote tecnológico específico para estas espécies acabou impossibilitando a viabilidade econômica da produção destes camarões. A partir do sucesso do cultivo da espécie exótica Litopenaeus vannamei no Nordeste, produtores da região Sul, principalmente de Santa Catarina, decidiram investir na atividade usando esta espécie. Segundo a EPAGRI, órgão de extensão do Estado de Santa Catarina, 800ha de viveiros estavam produzindo $L$. vannamei e a previsão para o final de 2004 é de praticamente dobrar a área de cultivo, chegando a 1500ha de viveiros.

Com os avanços da atividade de cultivo de camarão em Santa Catarina, alguns produtores rurais e investidores do Rio Grande do Sul começaram a se mobilizar com intuito de estabelecer a atividade na região.

Em função das características ambientais e pelos menores custos de implantação, o estuário da Lagoa dos Patos vem sendo a área preferida para implantação destes empreendimentos. Atualmente, a primeira fazenda produtora de camarões marinhos está localizada no município de São José do Norte, onde outras quatro fazendas aguardam autorização para serem instaladas, totalizando aproximadamente 125 hectares de viveiros. Em Rio Grande, município vizinho a São José do Norte, uma fazenda está em funcionamento e outra aguarda licença ambiental para iniciar o cultivo.
Paralelamente à instalação de fazendas de cultivo de camarões em viveiros, a Fundação Universidade Federal do Rio Grande, por meio da Estação Marinha de Aquacultura, também vem apoiando os pescadores artesanais da região no cultivo do camarão nativo Farfantepenaeus paulensis em estruturas de baixo custo, instaladas em áreas rasas do estuário.

\section{O ESTUÁRIO DALAGOADOS PATOS}

O estuário da Lagoa dos Patos (Figura 1), assim como outros, possui grande importância econômica e também ecológica (ASMUS \& TAGLIANI, 1998). Do ponto de vista econômico, as principais atividades desenvolvidas na região estuarina estão relacionadas ao porto, à produção industrial, à agricultura e à pesca, tanto industrial como artesanal (REIS \& DINCAO, 2000).

Em contraste com as atividades antrópicas exercidas na região está a importância ecológica deste ambiente, que é caracterizada por uma grande produção biológica (SEELIGER et al., 1998) e pela presença de muitas espécies de plantas e animais que em algum momento de seu ciclo de vida utilizam este ambiente para o seu desenvolvimento.

Nas águas do estuário da Lagoa dos Patos, observam-se grandes variações de temperatura e salinidade, que são determinadas principalmente pela ação dos ventos, pela temperatura do ar ambiente e pelo regime de chuvas atuantes no período (VILAS BOAS, 1990). Em função desta dinâmica, pode-se observar grande flutuação na composição química da água, que tende a variar em função da mudança de salinidade(NIENCHESKI \& WINDOM, 1994). Do ponto de vista da atividade de cultivo de camarões, sob condições climatológicas normais, a porção sul do estuário é a mais atraente para a atividade de cultivo, pois tende a apresentar um regime de águas salobras devido à maior influência das águas do oceano, condição ambiental determinante para o sucesso do cultivo dos camarões (WASIELESKY et al., 2003).

Outras características ambientais que tornam o estuário atrativo para o cultivo de camarões são a pequena variação de maré, que permite bombeamento constante ao longo do dia; a hidro-dinâmica moderada, o que facilita o posicionamento do sistema de bombeamento; e a fertilidade natural das águas, que favorece o desenvolvimento da produção primária nos viveiros.

\section{OUTROS FATORES QUE FAVORECEM A ATIVIDADE NAREGIÃO \\ Para determinar a viabilidade de um projeto de cultivo, além de levantar informações sobre as}




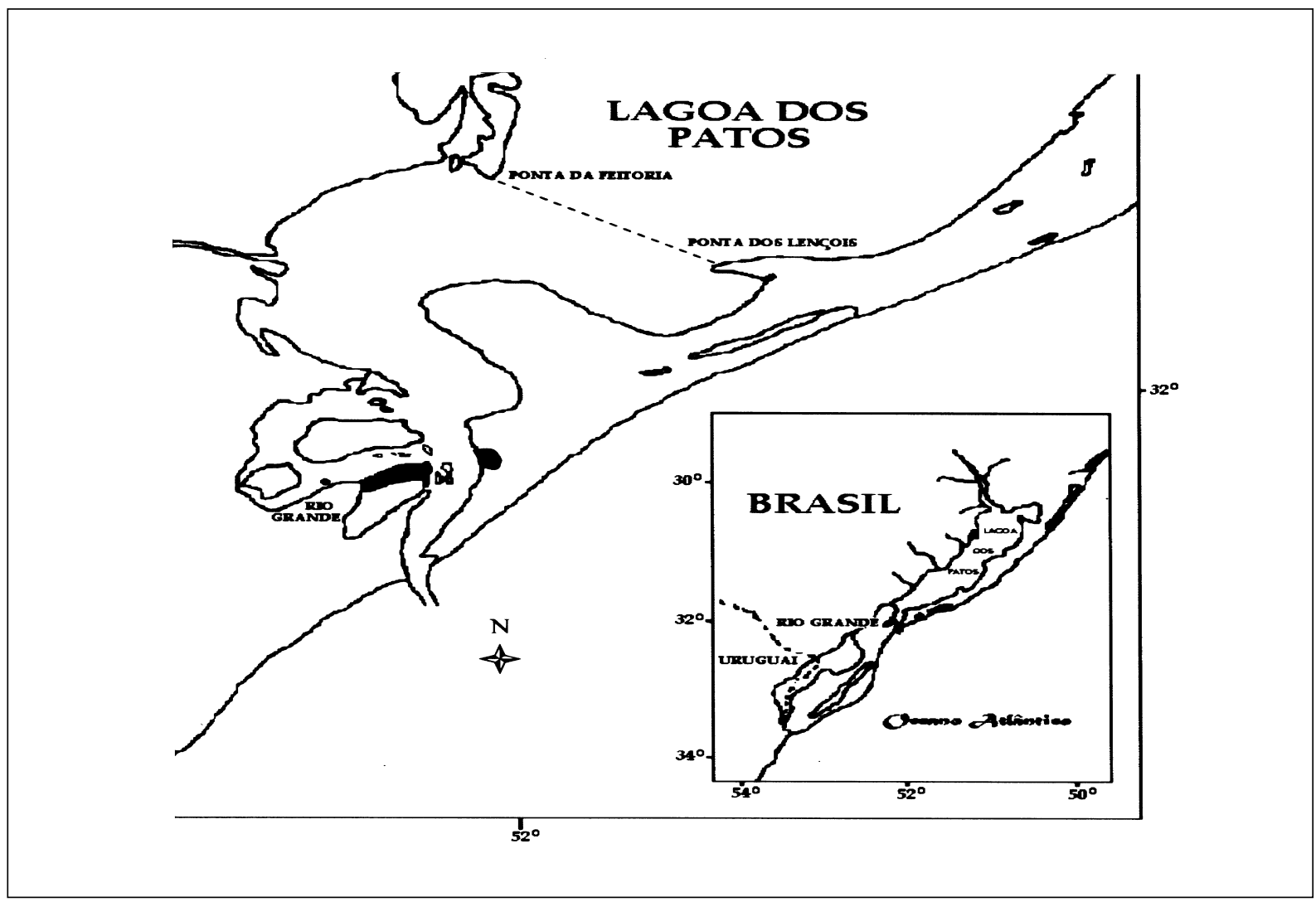

Figura 1 - Representação da Lagoa dos Patos (imagem menor) e do estuário (delimitado pela linha tracejada).

características ambientais do local de implantação, deve-se também considerar a infra-estrutura disponível e realizar um estudo de viabilidade econômica. Com base neste conjunto de informações, pode-se optar pela instalação ou não do empreendimento de cultivo.

No Estado do Rio Grande do Sul, já estão instaladas indústrias voltadas para a atividade de cultivo de camarões e que distribuem seus produtos para outras regiões do Brasil. Indústrias de ração para camarão, de bombas hidráulicas e de equipamentos auxiliares (comedores, comportas, caiaques e remos, entre outros) favorecem o desenvolvimento da atividade na região. Este favorecimento não é só referente ao menor preço do frete pago pelo produtor, mas também pela disponibilidade do produto.

Além desses fatores, na porção sul do Estado, pode-se considerar ainda o baixo preço da área junto ao estuário, o alcance abrangente da eletrificação rural, a presença de rodovias para chegada de insumos e escoamento do produto final, a disponibilidade de mão-de-obra especializada, o parque de indústrias de processamento de pescado e a presença de um dos mais importantes portos marítimos da América do Sul.
FORMAS DE CULTIVOEMPREGADAS NAREGIÃO

Vários são os sistemas de cultivo de camarões empregados no mundo, incluindo viveiros em terra, tanques em concreto, gaiolas flutuantes e fixas, e cercados. A forma de cultivo também pode ser diferenciada pela utilização ou não de alimento inerte e pelas taxas de renovação de água. Quanto maior for a densidade de estocagem dos organismos por área cultivada (sistema intensivo), maiores serão as taxas de renovação de água e a quantidade de alimento oferecido.

\section{Cultivo em viveiros}

No caso específico do estuário da Lagoa dos Patos, o cultivo de camarões em viveiros tem sido baseado na utilização de sistemas extensivos e semiintensivos. A solicitação das licenças de instalação junto ao governo estadual prevê densidades de estocagem de até 30 camarões $\mathrm{m}^{-2}$. Estabeleceu-se também a utilização de bandejas de alimentação, empregadas como forma de minimizar as perdas de alimento, diminuir o custo de produção e melhorar a qualidade das águas que saem dos viveiros. Normalmente são empregadas taxas de renovação 
diárias de até $5 \%$ do volume total dos viveiros. Este volume de água é destinado obrigatoriamente a uma bacia de sedimentação, que representa em torno de 7\% da área total dos viveiros da fazenda e que permite a diminuição significativa no aporte da matéria orgânica e de nutrientes para o meio ambiente (POERSCH, 2004).

\section{Cultivo em cercados:}

Os pescadores artesanais que realizam cultivo em cercados (Figura 2) trabalham com densidades variando entre 15 e 30 camarões por metro quadrado. Especial atenção tem sido dada pela universidade, no intuito de continuar trabalhando com a espécie nativa $\boldsymbol{F}$. paulensis nas estruturas de cultivo, já que, se fosse introduzida uma espécie exótica e ocorresse uma fuga dos cercados, seria possível estarse dando início a um sério problema ambiental. Por outro lado, busca-se a diminuição dos custos de instalação dos cercados com a utilização de material de baixo valor (por exemplo: bambu) para fixação da malha (poliéster revestida com PVC). Da mesma forma, estudos estão sendo realizados para substituir a ração, utilizada como alimento para os camarões por pescado (peixes e siris) não comercializado pelos próprios pescadores e ainda por subprodutos da pesca, como a cabeça de camarão e de peixes.

\section{LEGISLAÇÃOE REGULAMENTAÇÃO}

Segundo VALENTI (2000), a aquacultura está embasada em três pilares, que seriam o ambiental, o econômico e o social. A interação entre estes setores

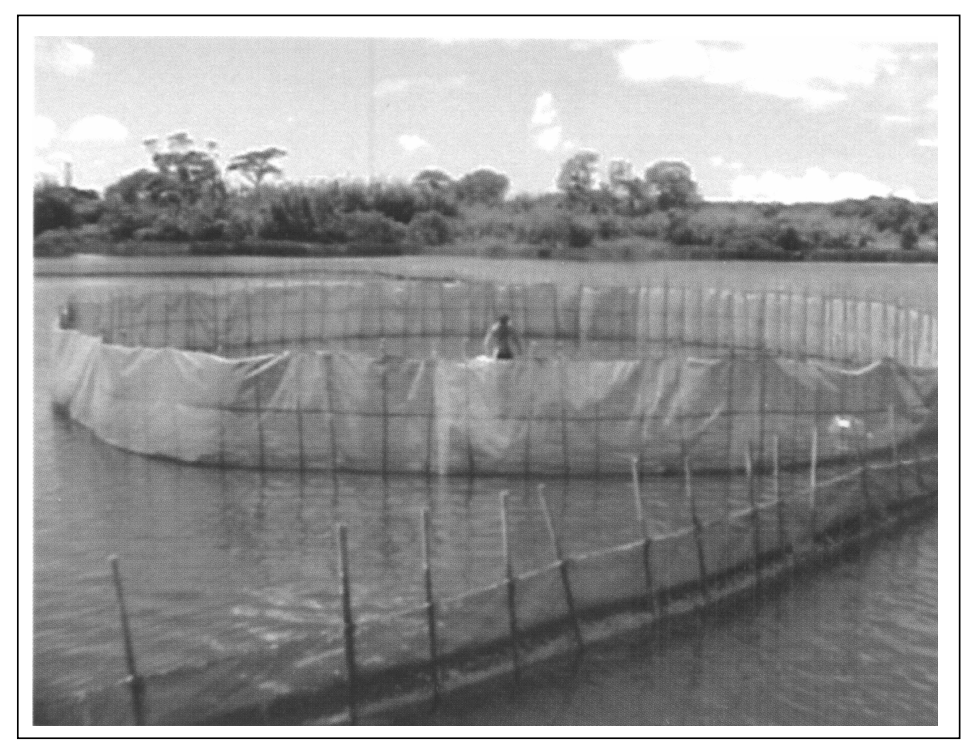

Figura 2 - Cercado para cultivo do camarão nativo $\boldsymbol{F}$. paulensis no estuário da Lagoa dos Patos, RS. é que vai determinar a sustentabilidade da atividade. No entanto, quando existem diferentes atores, com interesses distintos, esta dinâmica relação pode acarretar conflitos, que deveriam ser mediados pelo poder público. ASSAD \& BURSZTYN (2000) salientam que devem ser feitos arranjos institucionais, em nível federal, estadual e municipal, para regular ou ordenar o desenvolvimento da atividade. No Estado do Rio Grande do Sul, a primeira licença de produção de camarões marinhos foi solicitada em 1999 e emitida pelo órgão ambiental (FEPAM - Fundação Estadual de Proteção Ambiental) em 2002. A demora das autoridades em autorizar o início da atividade foi necessária para regulamentar a introdução do camarão branco do pacífico (L. vannamei) e determinar sob quais condições seria permitido o cultivo desta espécie. Desta forma, as fazendas de cultivo no Rio Grande do Sul devem, dentre os principais requerimentos, assegurar que: a espécie cultivada não tenha acesso ao ambiente natural, por meio de um efetivo conjunto de telas de proteção; os taludes dos viveiros sejam construídos obedecendo as normas de engenharia da aquacultura; a água que sai dos viveiros deve sofrer tratamento primário, passando necessariamente por uma bacia de sedimentação (Figura 3); áreas de proteção ambiental, como dunas, mata nativa e áreas alagadas (marismas) sejam mantidas preservadas (Figura 3); a área de recuo próxima à margem da lagoa, a partir do limite entre a zona de marisma e a terrestre, não seja inferior a 100 metros (Figura 3).

A adoção dessas normas por parte do órgão ambiental estadual tem provocado as mais diversas reações. Por um lado, os municípios buscam incentivar a atividade de cultivo e desta forma reaquecer a economia local, mas, por outro, os requerimentos impostos pelo órgão ambiental aumentam os custos de investimento, muitas vezes impossibilitando a implantação de empreendimentos, principalmente por parte de pequenos produtores. Nesse sentido, o posicionamento do órgão ambiental estadual (FEPAM) frente aos cultivos de camarões é um pouco incoerente, já que coloca pequenos e grandes produtores em uma mesma classificação, quando comparado com as normas do Conselho Nacional do Meio Ambiente CONAMA. Na tabela 1, pode-se observar como o órgão ambiental estadual classifica hoje os empreendimentos em relação à classificação do CONAMA. 


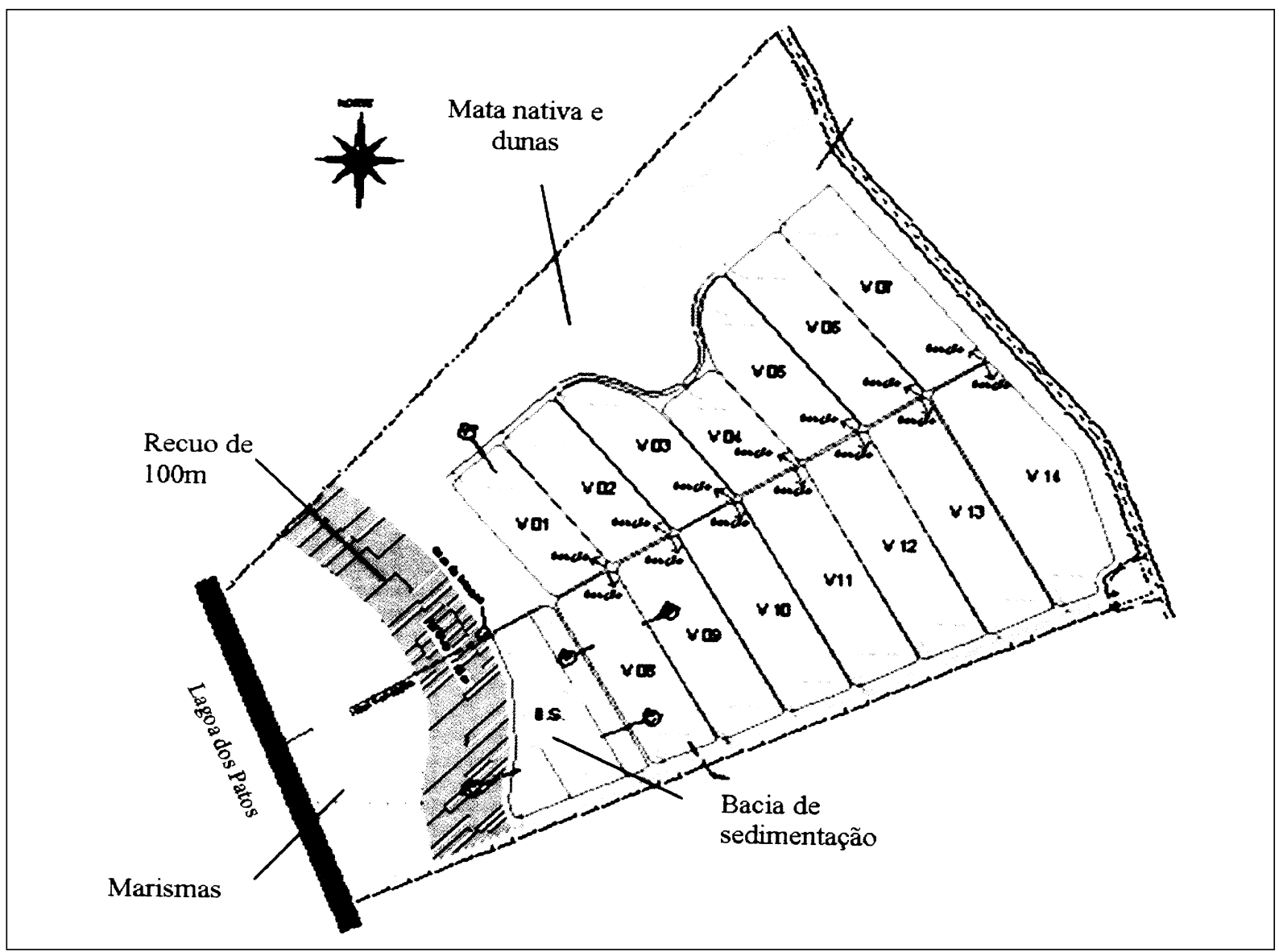

Figura 3 - Distribuição dos viveiros de cultivo, da bacia de sedimentação e das áreas de preservação ambiental (mata nativa, dunas e marismas). Projeto elaborado pelo grupo de pesquisadores da Estação Marinha de Aquacultura - FURG.

Seria lógica a adoção de medidas diferenciando os produtores em função da área utilizada para cultivo, já que quanto menor a área de cultivo menor o potencial poluidor. No entanto, a classificação empregada pelo Estado do Rio Grande do Sul é demasiadamente conservadora. Se por um lado o governo estadual é bastante rigoroso com a questão de aplicação das normas estabelecidas, por outro peca pela falta de estudo para determinação das áreas mais propícias para a instalação das fazendas. No estuário da Lagoa dos Patos, são observados diferentes ambientes, que apresentam características peculiares. Por exemplo, a zona próxima ao canal de acesso possui hidrodinâmica que difere das enseadas, e estas também diferem entre si. Por isso, seria de extrema importância a realização do zoneamento da região, enquanto a atividade ainda se encontra em fase inicial de desenvolvimento. Dessa forma, poderiam ser evitados ou minimizados problemas de impacto ambiental e de alteração na produção de peixes e crustáceos que utilizam o estuário como criadouro.

Com relação aos cultivos em cercados e gaiolas, somente em 2004 o governo federal, por meio da instrução normativa $n^{\circ} 6$, estabeleceu regras para uso de áreas aquícolas da união com finalidade de aquacultura. Até então, as unidades de cultivo montadas no estuário da Lagoa dos Patos vinham sendo subvencionadas pelos diferentes órgãos de apoio à pesquisa, sendo o gerenciamento da atividade realizado pela Estação Marinha de Aquacultura da FURG

\section{FUTURO DA ATIVIDADE}

Os cultivos de camarões podem ser uma alternativa viável para o desenvolvimento econômico e social da região estuarina da Lagoa dos Patos. A atividade hoje apresenta uma boa rentabilidade, com retorno do investimento estimado em aproximadamente 
Tabela 1 - Comparação da classificação das fazendas de cultivo de camarão no Estado do Rio Grande do Sul de acordo com a Fundação Estadual de Proteção Ambiental (FEPAM) e com o Conselho Nacional do Meio Ambiente (CONAMA).

\begin{tabular}{llllll}
\hline Área de cultivo (ha) & $0-2$ & $2-5$ & $5-10$ & $10-50$ & $>50$ \\
\hline FEPAM & Pequena & Média & Grande & Excepcional & Excepcional \\
CONAMA & Pequena & Pequena & Pequena & Média & Grande \\
\hline
\end{tabular}

três anos, considerando apenas uma safra por ano. Estudos estão sendo realizados com a introdução de berçários no sistema de cultivo e os resultados preliminares apontam para a possibilidade de uma segunda safra na região, o que tornaria o investimento ainda mais atrativo.

Do ponto de vista social, a atividade pode absorver boa parte da mão de obra ociosa da região. Estima-se que fazendas de pequeno porte (até $10 \mathrm{ha}$ ), utilizem de 1,3 a 1,4 funcionários por cada hectare cultivado (COSTA \& SAMPAIO, 2004). No mesmo estudo, considerando toda a cadeia produtiva (laboratórios de produção de pós-larvas - fazendas de cultivo - processamento), os autores determinaram uma relação de praticamente 2,0 empregos diretos por cada hectare cultivado, o que supera setores tradicionais do agronegócio.

Tomando como exemplo o município de São José do Norte (RS), com uma população de 24.795 habitantes e uma economia praticamente estagnada (crescimento negativo de 4,71\% nos últimos 5 anos), estima-se que os 125 hectares das fazendas de camarão gerem aproximadamente 180 empregos nas propriedades, sem considerar o restante da cadeia produtiva. Estimando um custo de implantação de R\$ 25.000 por hectare de viveiro, tem-se aplicado no município aproximadamente $\mathrm{R} \$ 3.125 .000$, que, somados a R\$ 600.000 empregados na construção de casas e galpões (infra-estrutura básica de gerenciamento), totalizam R\$3.725.000 aplicados no município. Com esta área cultivada, as projeções para o lucro bruto indicam um montante em torno de $\mathrm{R} \$ 2.500 .000$ por ciclo, podendo alcançar até $\mathrm{R} \$ 5.000 .000$ por ano, no caso da obtenção de dois ciclos. Logicamente, este recurso não é totalmente voltado para a economia local; no entanto, parte fica disponível, por meio da cobrança de impostos, e parte vai estimular a geração de novos comércios na cidade, como venda e manutenção de bombas hidráulicas, material elétrico, aeradores, caiaques, ração e serviços como terraplanagem, transporte de camarão, fabrico de gelo e outros serviços especializados.

Pensando em um futuro próximo, estima-se que as características locais permitiriam a expansão da área cultivada em torno de $1.000 \%$, o que significaria a geração de um rendimento de R \$ 50.000.000, potencialmente disponíveis ao mercado.
Para os cultivos em cercados, os montantes não são tão expressivos quanto os apresentados anteriormente; no entanto, o impacto social seria mais importante (HOLZ, 2001). O estuário representa uma área de aproximadamente 100.000 hectares, dos quais $1 \%$ poderia ser utilizado para o cultivo em cercados de 0,3 hectares. Este 1\% da área do estuário equivale a 1.000 hectares, nos quais poderiam ser distribuídos 3.000 unidades de cultivo, praticamente um cercado por família de pescador artesanal. Como os estudos realizados indicam, em anos normais, com uma produção mínima de $400 \mathrm{~kg}$ de camarão por cercado, o pescador poderia ter uma renda adicional de $\mathrm{R} \$ 4.000$ por ano, ou R\$333,33 por mês. Considerando que a renda média do pescador é inferior ao salário mínimo, este adicional poderia melhorar sensivelmente as condições de vida destas famílias (WASIELESKY, 2000).

Do ponto de vista ambiental, a implantação de cultivos de maneira descontrolada pode gerar sérios problemas ao ambiente e aos próprios cultivos. Cabe ao governo, em conjunto com as instituições de pesquisa e com as organizações não-governamentais, estabelecer normas que permitam o estabelecimento da atividade, mantendo o equilíbrio sócio-econômico, sem que haja prejuízo do ponto de vista ambiental. Da mesma forma, seria interessante, para toda a cadeia produtiva, que as políticas de desenvolvimento gerenciadas pelo governo estimulassem a adoção de normas de conduta ou de manejo por parte dos produtores.

Com a adoção de normas de conduta coerentes e responsáveis, estaríamos preservando o futuro da atividade, melhorando o quadro sócioeconômico da região e, ao mesmo tempo, mantendo o ambiente com um menor grau de comprometimento.

\section{REFERÊNCIAS}

ABCC. A carcinocultura brasileira. Revista da ABCC, v.1, p.30-45, 2004.

ASMUS, M.L.; TAGLIANI, P.R. Considerações sobre manejo ambiental. In: SEELIGER et al. Os ecossistemas costeiro e marinho do extremo sul do Brasil. Rio Grande, 1998. 326p.

Ciência Rural, v.36, n.4, jul-ago, 2006. 
ASSAD, L.T.; BURSZTYN, M. Aqüicultura no Brasil: bases para o desenvolvimento sustentável. Brasilia: $\mathrm{CNPq} /$ Ministério de Ciência e Tecnologia, 2000. 33-72p.

COSTA, E.; SAMPAIO, Y. Direct and indirect job generation in the farmed shrimp chain. Aquaculture Economics and Management, v.8, n.3-4, p.143-156, 2004.

FAO. Aquaculture production statistics 1987-2000. Fishery statistics: aquaculture production, 2002. V.90/2.

HOLZ, R.E. Análise econômica preliminar entre a pesca e o cultivo do camarão-rosa Farfantepenaeus paulensis no estuário da Lagoa dos Patos. 2001. 48f. Monografia (Especialização) - Fundação Universidade Federal do Rio Grande, Rio Grande, RS.

NIENCHESKI, L.F.; WINDOM, H.L. Distribution of particulate trace metal in Patos Lagoon estuary (Brazil). Marine Pollution Bulletin, v.28, n.2, p.96-102, 1994.

POERSCH, L.H. Aquacultura no estuário da Lagoa dos Patos e sua influencia sobre o meio ambiente. 2004. 147f. Tese (Doutorado em Oceanografia Biológica) Fundação Universidade Federal do Rio Grande, Rio Grande, RS.

REIS, E.G.; D’INCAO, F. The present status of artisanal fisheries of extreme Southern Brazil: an effort towards community- based management. Ocean \& Coastal Management, v.43, p.585-595, 2000, 48p.

SEELIGER, U. et al. Fluxo de energia e habitats no estuário da Lagoa dos Patos. In: SEELIGER et al. Os ecossistemas costeiro e marinho do extremo sul do Brasil. Rio Grande, 1998. 326p.

VALENTI, W.C. Aqüicultura no Brasil: bases para o desenvolvimento sustentável. Brasília: CNPq/Ministério de Ciência e Tecnologia, 2000. p.25-32.

VILAS BOAS, D.F. Distribuição e comportamento dos sais nutrientes, elementos maiores e metais pesados na Lagoa dos Patos, RS. 1990. 112f. Tese (Mestrado em Oceanografia Biológica) - FURG, Rio Grande, RS.

WASIELESKY, W. Cultivo do camarão rosa Farfantepenaeus paulensis (Decapoda, Penaeidae) no estuário da Lagoa dos Patos: efeitos de parâmetros ambientais e manejo de cultivo. 2000. 229f. Tese (Doutorado em Oceanografia Biológica) - Fundação Universidade Federal do Rio Grande, Rio Grande, RS.

WASIELESKY, W. et al. The effect of temperature, salinity and nitrogen products on food consumption of pink shrimp Farfantepenaeus paulensis. Brazilian Archives of Biology and Technology, v.46, n.1, p.135-141, 2003. 